\title{
Quasi-i-Boolean algebras vs. quasi-m Boolean algebras
}

\author{
Afrodita Iorgulescu \\ E-mail: afrodita.iorgulescu@ase.ro
}

In memoriam: Vlad Boicescu

\begin{abstract}
We introduce the quasi-i-Boolean algebras and the quasi-m Boolean algebras and we prove their categorically equivalence.

Keywords: Quasi-Wajsberg algebra, Quasi-MV algebra, Quasi-i-Boolean algebra, Quasi-m Boolean algebra, Wajsberg algebra, MV algebra, I-Boolean algebra, Boolean algebra
\end{abstract}

\section{Introduction}

The departure point for the research was the introducing of the quasi- $M V$ algebras in 2006 [15], as generalizations of MV algebras, following an investigation into the foundations of quantum computing (see [4]); since then, many papers investigated them $[17,1,6,14]$. Then, the introducing of the quasiWajsberg algebras in 2010 [2], as generalizations of Wajsberg algebras; they are categorically equivalent to quasi-MV algebras, just as Wajsberg algebras are categorically equivalent to MV algebras.

In paper [10], starting from the quasi-Wajsberg algebras, whose regular algebras are the Wajsberg algebras, we have introduced a theory of quasi-algebras (of logic) vs. a theory of regular algebras (of logic), in the commutative case. We then have developed the theory in the preprints $[11,12]$. In [12], we have introduced the quasi-i-Boolean algebras, whose regular algebras are the i-Boolean algebras.

In [13], we have noted that, in fact, there are two kinds of "quasi"-generalizations, that we have called: "quasi-" and "quasi-m", corresponding to the two kinds of algebras: $M$ algebras (inside the commutative algebras of logic) and commutative unital magmas, respectively ("m" comes from magma). Thus, (in [13] is the non-commutative case) we have generalized the $M$ algebras to quasi- $M$ algebras, as the most general quasi-algebras, by generalizing the principal, defining property (M) to (q-M), and we have generalized the commutative unital magmas to commutative quasi-m unital magmas, as the most general quasi-m algebras, by generalizing the principal, defining property (U) to (qm-U).

The Wajsberg algebra and the $i$-Boolean algebra belong to the "world of commutative algebras of logic", more precisely to the class of $M$ algebras; the $M V$ algebra and the Boolean algebra belong to the "world of commutative algebras", more precisely to the class of commutative unital magmas.

This small paper is organized as follows. In Section 2 , we recall some basic things about regular algebras (structures), from [10], and about regular-m algebras (structures), from [13]; we recall the i-Boolean algebras and the Boolean algebras, and their categorically equivalence. In Section 3, we recall some basic things about quasi-algebras (quasi-structures), from [10], and about quasi-m algebras (structures), from [13]; we introduce the quasi-i-Boolean algebras and the quasi$\mathrm{m}$ Boolean algebras and we prove their categorically equivalence (the main result); finally, we present some examples.

\section{Regular algebras vs. regular-m algebras}

\subsection{Introduction to a theory of regular algebras (structures)}

Let $\mathcal{A}=(A, \rightarrow, 1)$ be an algebra of type $(2,0)$ through this paper, where a binary relation $\leq$ can be defined by: $x \leq y \stackrel{\text { def. }}{\Longleftrightarrow} x \rightarrow y=1$.

Equivalently, let $\mathcal{A}=(A, \leq, \rightarrow, 1)$ be a structure, where $\leq$ is a binary relation on $A, \rightarrow$ is a binary operation (an implication) on $A$ and $1 \in A$, all connected by the equivalence: $x \leq y \Longleftrightarrow x \rightarrow y=1$.

Consider the property: for all $x \in A$, (M) $1 \rightarrow x=x$.

\section{Definitions 2.1 [10]}

(1) The algebra $\mathcal{A}=(A, \rightarrow, 1)$ (structure $\mathcal{A}=$ $(A, \leq, \rightarrow, 1))$ is called regular, if it satisfies the property $(\mathrm{M})$.

(1') Any algebra (structure) $\mathcal{A}^{\prime}=(A, \sigma)$ whose signature $\sigma$ contains $\rightarrow, 1(\leq, \rightarrow, 1$, respectively) is also 
called regular, if it satisfies (M).

(1") Any algebra (structure) $\mathcal{A}^{\prime \prime}=(A, \tau)$ which is term equivalent to a regular algebra (structure) $\mathcal{A}^{\prime}=$ $(A, \sigma)$, is also called regular.

An $M$ algebra is an algebra $\mathcal{A}=(A, \rightarrow, 1)$ verifying (M) [13]. Note that the class of $M$ algebras is the largest class of regular algebras. Other examples of regular algebras are the BCI, BCK algebras, the Hilbert algebras, the commutative implicative-groups [13], the Wajsberg algebras etc.

Let us consider the following subsets of $A[10]$ : $U \stackrel{\text { def. }}{=}\{x \rightarrow y \mid x, y \in A\}, \quad V \stackrel{\text { def. }}{=}\{1 \rightarrow x \mid x \in A\}$, $V_{M} \stackrel{\text { def. }}{=}\{x \in A \mid x \stackrel{(M)}{=} 1 \rightarrow x\}$.

By (M), we have that: $V_{M}=V=U=A$, and this is the basic, definable property of regular algebras (structures). In the theory of regular algebras (structures), we determine which properties depend on (M) (are proved by $(\mathrm{M})$ ) and which are independent of $(\mathrm{M})$; this is the necessary step to develop further the theory of quasi-algebras (quasi-structures).

\subsubsection{Implicative-Boolean algebras}

There are many equivalent definitions of Boolean algebras (see [16]). We recall here the definition introduced in 2009 [8] and presented also in [3], motivated by the axioms system of the classical propositional logic:

(G1) $\varphi \rightarrow(\psi \rightarrow \varphi)$,

(G2) $(\varphi \rightarrow(\psi \rightarrow \chi)) \rightarrow((\varphi \rightarrow \psi) \rightarrow(\varphi \rightarrow \chi))$,

(G3) $(\neg \psi \rightarrow \neg \varphi) \rightarrow(\varphi \rightarrow \psi)$.

An implicative-Boolean algebra, or an $i$-Boolean algebra for short, is an algebra

$$
\mathcal{A}=\left(A, \rightarrow,{ }^{-}, 1\right)
$$

of type $(2,1,0)$ verifying: for all $x, y, z \in A$,

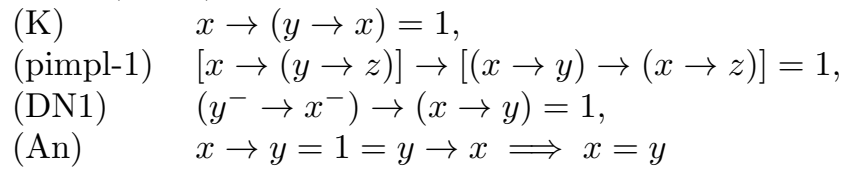
(Antisymmetry).

Let $\mathcal{A}$ be an i-Boolean algebra. The following properties hold, among many others [12]:

(M) $1 \rightarrow x=x$;

(Re) (Reflexivity) $x \rightarrow x=1$,

(L) (Last element) $x \rightarrow 1=1$,

(F) (First element) $0 \rightarrow x=1$,

(B) $(y \rightarrow z) \rightarrow[(x \rightarrow y) \rightarrow(x \rightarrow z)]=1$,

(*) $y \rightarrow z=1 \Longrightarrow(x \rightarrow y) \rightarrow(x \rightarrow z)=1$,

$(* *) y \rightarrow z=1 \Longrightarrow(z \rightarrow x) \rightarrow(y \rightarrow x)=1$,

(D) $y \rightarrow[(y \rightarrow x) \rightarrow x]=1$,

(Ex) (Exchange) $x \rightarrow(y \rightarrow z)=y \rightarrow(x \rightarrow z)$;

(Tr) (Trans.) $x \rightarrow y=1=y \rightarrow z \Longrightarrow x \rightarrow z=1$,

(impl) (implicative) $(x \rightarrow y) \rightarrow x=x$;

(Neg) $x^{-}=x \rightarrow 0$
(DN) (Double negation) $\left(x^{-}\right)^{-}=x$;

(Neg1-0) $1^{-}=0$,

$(\mathrm{Neg} 0-1) 0^{-}=1$;

(Neg2) $x \rightarrow y=1 \Longrightarrow y^{-} \rightarrow x^{-}=1$,

(Neg3) $y \rightarrow x^{-}=x \rightarrow y^{-}$;

(Neg6) $x \rightarrow x^{-}=x^{-}$,

(DN2) $x \rightarrow y=y^{-} \rightarrow x^{-}$,

(DN6) $x^{-} \rightarrow x=x$.

Note that an i-Boolean algebra is a regular algebra, since (M) holds.

\subsection{Introduction to a theory of regular- $\mathbf{m}$ algebras (structures)}

Let $\mathcal{A}=(A, \odot, 1)$ be a commutative algebra of type $(2,0)$ or let $\mathcal{A}=(A, \leq, \odot, 1)$ be a commutative structure, through this paper, where commutative means $x \odot y=y \odot x$, for all $x, y \in A$.

Consider the property: for all $x \in A$,

(U) $1 \odot x=x$.

\section{Definitions 2.2 [13]}

(1m) The commutative algebra $\mathcal{A}=(A, \odot, 1)$ of type $(2,0)$ (structure $\mathcal{A}=(A, \leq, \odot, 1)$ ) is called regular- $m$, if it satisfies the property $(\mathrm{U})$.

(1'm) Any commutative algebra (structure) $\mathcal{A}^{\prime}=$ $(A, \sigma)$ whose signature $\sigma$ contains $\odot, 1(\leq, \odot, 1$, respectively) is also called regular- $m$, if it satisfies (U).

(1"m) Any algebra (structure) $\mathcal{A}^{\prime \prime}=(A, \tau)$ which is term equivalent to a regular-m algebra (structure) $\mathcal{A}^{\prime}=(A, \sigma)$, is also called regular-m.

A commutative unital magma is a commutative algebra $\mathcal{A}=(A, \odot, 1)$ verifying (U). Note that their class is the largest class of regular-m algebras $(" \mathrm{~m} "$ comes from magma). Other examples of regular-m algebras are the commutative monoids, the commutative groups, the MV algebras etc.

Let us consider the following subset of $A[13]$ :

$$
V_{U} \stackrel{\text { def. }}{=}\{x \in A \mid x \stackrel{(U)}{=} 1 \odot x\} \subseteq A \text {. }
$$

Note that, if property (U) holds, then $A \subseteq V_{U}$, hence $V_{U}=A$, and this is the basic, definable property of regular-m algebras (structures). In the theory of regular-m algebras (structures), we determine which properties depend on (U) (are proved by $(\mathrm{U})$ ) and which are independent of $(\mathrm{U})$; this is the necessary step to develop further the theory of quasi-m algebras (structures).

\subsubsection{Boolean algebras}

Boolean algebras were introduced in 1854 by George Boole. The most used definition is as a complemented, bounded, distributive lattice, namely: 
A Boolean algebra is an algebra

$$
\mathcal{A}=\left(A, \wedge, \vee,^{-}, 0,1\right)
$$

of type $(2,2,1,0,0)$ verifying: for all $x, y, z \in A$,

(mWid)

$x \wedge x=x$

(mVid)

$x \vee x=x$

$(\mathrm{mWcomm}) \quad x \wedge y=y \wedge x$,

$(\mathrm{mVcomm}) \quad x \vee y=y \vee x$

$(\mathrm{mWass}) \quad x \wedge(y \wedge z)=(x \wedge y) \wedge z$,

$(\mathrm{mVass}) \quad x \vee(y \vee z)=(x \vee y) \vee z$;

$(\mathrm{mAbs} 1) \quad x \wedge(x \vee y)=x$,

$(\mathrm{mAbs} 2) \quad x \vee(x \wedge y)=x$;

$(\mathrm{mDis} 1) \quad x \wedge(y \vee z)=(x \wedge y) \vee(x \wedge z)$,

$(\mathrm{mDis} 2) \quad x \vee(y \wedge z)=(x \vee y) \wedge(x \vee z)$;

$(\mathrm{mUW}) \quad 1 \wedge x=x$,

$(\mathrm{mUV}) \quad 0 \vee x=x$

$(\mathrm{mWx}) \quad x \wedge x^{-}=0$

$(\mathrm{mVx}) \quad x \vee x^{-}=1$.

Note that a Boolean algebra (that can be defined equivalently as an algebra $\left(A, \wedge,{ }^{-}, 1\right)$ of type $\left.(2,1,0)\right)$ is a regular-m algebra, since (mUW), and the dual (mUV), hold.

\subsection{Implicative-Boolean algebras vs. Boolean algebras}

The i-Boolean algebras are categorically equivalent to the Boolean algebras just as the Wajsberg algebras are categorically equivalent to the MV algebras, namely we have:

\section{Theorem 2.3 [3]}

(1) Let $\mathcal{A}=\left(A, \rightarrow,{ }^{-}, 1\right)$ be an $i$-Boolean algebra. Define $\Phi(\mathcal{A}) \stackrel{\text { def. }}{=}(A, \wedge, \vee,-, 0,1)$ as follows: for every $x, y \in A, x \wedge y \stackrel{\text { def. }}{=}\left(x \rightarrow y^{-}\right)^{-}$, $x \vee y \stackrel{\text { def. }}{=}\left(x^{-} \wedge y^{-}\right)^{-}=x^{-} \rightarrow y, \quad 0 \stackrel{\text { def. }}{=} 1^{-}$.

Then, $\Phi(\mathcal{A})$ is a Boolean algebra.

(1') Conversely, let $\mathcal{A}=\left(A, \wedge, \vee,^{-}, 0,1\right)$ be a Boolean algebra.

Define $\Psi(\mathcal{A}) \stackrel{\text { def. }}{=}(A, \rightarrow,-, 1)$ as follows: for every $x, y \in A, x \rightarrow y \stackrel{\text { def. }}{=}\left(x \wedge y^{-}\right)^{-}=x^{-} \vee y$.

Then, $\Psi(\mathcal{A})$ is an $i$-Boolean algebra.

(2) The mappings $\Phi$ and $\Psi$ are mutually inverse.

\section{Quasi-algebras vs. quasi-m algebras}

\subsection{Introduction to a theory of quasi- algebras (quasi-structures)}

Let $\mathcal{A}=(A, \rightarrow, 1)$ be an algebra of type $(2,0)$ or, equivalently, let $\mathcal{A}=(A, \leq, \rightarrow, 1)$ be a structure. Consider the properties: for all $x, y \in A$,
$(\mathrm{q}-\mathrm{M}) 1 \rightarrow(x \rightarrow y)=x \rightarrow y$,

(M1) $1 \rightarrow 1=1$.

Definitions 3.1 [10]

(q1) The algebra $\mathcal{A}=(A, \rightarrow, 1)$ (structure $\mathcal{A}=$ $(A, \leq, \rightarrow, 1))$ is called quasi-algebra (quasi-structure, respectively), if it satisfies the properties (q-M) and (M1).

(q1') Any algebra (structure) $\mathcal{A}^{\prime}=(A, \sigma)$ whose signature $\sigma$ contains $\rightarrow, 1(\leq, \rightarrow, 1$, respectively) is also called quasi-algebra (quasi-structure), if it satisfies (q-M) and (M1).

(q1") Any algebra (structure) $\mathcal{A}^{\prime \prime}=(A, \tau)$ which is term equivalent to a quasi-algebra (quasi-structure) $\mathcal{A}^{\prime}=(A, \sigma)$, is also called quasi-algebra (quasistructure).

A quasi- $M$ algebra is an algebra $\mathcal{A}=(A, \rightarrow, 1)$ verifying (q-M) and (M1) [13]. Note that their class is the largest class of quasi-algebras. Other examples of quasi-algebras are the quasi-BCI, the quasiBCK algebras [10], the quasi-Hilbert algebras [11], the commutative quasi-implicative-groups [13], the quasiWajsberg algebras [2].

Remark 3.2 [10] (q-M) is different of (M) if and only if $V_{M}=V=U \subset A$, and this is the basic, definable property of quasi-algebras (quasi-structures).

For every quasi-algebra (quasi-structure) $\mathcal{A}$, the subset $V_{M}=V=U$ of $A$ will be called the regular set of $\mathcal{A}$ and will be denoted by $R(A)$ :

$$
R(A) \stackrel{\text { def. }}{=} V_{M}=V=U \subseteq A \text {. }
$$

The quasi-algebra (quasi-structure) $\mathcal{A}$ is called $q$ proper, if $R(A) \neq A$ (i.e. (M) $\Longleftrightarrow(\mathrm{q}-\mathrm{M})$ ); otherwise, $\mathcal{A}$ is a regular algebra (structure). The elements of $R(A)$ are called the regular elements of $A$, the elements of $A \backslash R(A)$ are called the quasi-elements of $A$.

Theorem $3.3[10]$ Let $\mathcal{A}=(A, \rightarrow, 1)$ be a q-proper quasi-algebra. Then, $\mathcal{R}(\mathcal{A})=(R(A), \rightarrow, 1)$ is a regular algebra.

In a q-proper quasi-algebra, we shall call quasiproperty, a property that is dependent of (q-M), and we shall call regular property, a property that is independent of (q-M).

\subsubsection{Quasi-implicative-Boolean algebras}

We have introduced the notion of quasi-implicativeBoolean algebra in the preprint [12], as follows:

A quasi-implicative-Boolean algebra, or a quasi-iBoolean algebra for short, is an algebra

$$
\mathcal{A}=\left(A, \rightarrow,^{-}, 1\right)
$$


of type $(2,1,0)$ verifying: for all $x, y, z \in A$,

(K) $x \rightarrow(y \rightarrow x)=1$,

(pimpl-1) $\quad[x \rightarrow(y \rightarrow z)] \rightarrow[(x \rightarrow y) \rightarrow(x \rightarrow z)]=1$, (DN1) $\left(y^{-} \rightarrow x^{-}\right) \rightarrow(x \rightarrow y)=1$,

(q-An) $x \rightarrow y=1=y \rightarrow x \Longrightarrow 1 \rightarrow x=1 \rightarrow y$ (quasi-Antisymmetry),

$(\mathrm{q}-\mathrm{M}) \quad 1 \rightarrow(x \rightarrow y)=x \rightarrow y$,

$(\mathrm{DN}) \quad\left(x^{-}\right)^{-}=x$,

$(q-\overline{M(1 \rightarrow x)}) \quad 1 \rightarrow(1 \rightarrow x)^{-}=(1 \rightarrow x)^{-}$.

Note that a quasi-i-Boolean algebra is a quasialgebra, since (q-M) and (M1) hold. Indeed, by [10], $x \rightarrow 1 \stackrel{(q-M)}{=} 1 \rightarrow(x \rightarrow 1) \stackrel{(K)}{=} 1$, hence (L) holds and (L) implies (M1). Note also that, if (M) holds, then any quasi-i-Boolean algebra is an i-Boolean algebra.

Theorem 3.4 [12] Let $\mathcal{A}=\left(A, \rightarrow,{ }^{-}, 1\right)$ be a q-proper quasi-i-Boolean algebra. Then,

$$
\mathcal{R}(\mathcal{A})=\left(R(A), \rightarrow^{-},{ }^{-}, 1\right)
$$

is an i-Boolean algebra.

The quasi-i-Boolean algebras verify the regular properties: (Re), (L), (F), (B), (*), (**), (D), (Ex), (Tr), (Neg1-0), (Neg0-1), (Neg2), (Neg3), (DN2), among others [12], and the following quasi-properties, among others [12]:

(q-I1) $x \rightarrow y=(1 \rightarrow x) \rightarrow y$,

(q-I2) $x \rightarrow y=x \rightarrow(1 \rightarrow y)$;

(q-impl) (quasi-implicative) $(x \rightarrow y) \rightarrow x=1 \rightarrow x$;

$(\mathrm{q}-\mathrm{Neg}) \quad x \rightarrow 0=1 \rightarrow x^{-}=(1 \rightarrow x)^{-}$,

( $\alpha) \quad x \rightarrow 0=1 \rightarrow x^{-},(\beta) \quad 1 \rightarrow x^{-}=(1 \rightarrow x)^{-}$;

$(\mathrm{q}-\mathrm{Neg} 6) x \rightarrow(1 \rightarrow x)^{-}=(1 \rightarrow x)^{-}$,

$(\mathrm{q}-\mathrm{DN} 6)(1 \rightarrow x)^{-} \rightarrow x=1 \rightarrow x$.

Proposition 3.5 Let $\left(A, \rightarrow,{ }^{-}, 1\right)$ be a

quasi-i-Boolean algebra. Define, for all $x, y \in A$,

$x \wedge y \stackrel{\text { def. }}{=}\left(x \rightarrow y^{-}\right)^{-}, x \vee y \stackrel{\text { def. }}{=}\left(x^{-} \wedge y^{-}\right)^{-}=x^{-} \rightarrow y$, $x \leq y \stackrel{\text { def. }}{\Longleftrightarrow} x \rightarrow y=1$.

Then, we have: for all $x, y, z \in A$,

$(W E x)(x \wedge y) \rightarrow z=x \rightarrow(y \rightarrow z)$,

(j) $x \wedge y \leq x \leq x \vee y$

(jj) $x \leq y$ and $a \leq b$ imply $x \wedge a \leq y \wedge b, x \vee a \leq y \vee b$.

\subsection{Introduction to a theory of quasi-m algebras (structures)}

Let $\mathcal{A}=(A, \odot, 1)$ be a commutative algebra or let $\mathcal{A}=(A, \leq, \odot, 1)$ be a commutative structure. Let us consider the following properties: for all $x, y \in A$,

$(\mathrm{qm}-\mathrm{U}) \quad 1 \odot(x \odot y)=x \odot y$,

(U1) $1 \odot 1=1$.

Definitions 3.6 [13]

(qm1) The commutative algebra $\mathcal{A}=(A, \odot, 1)$ (structure $\mathcal{A}=(A, \leq, \odot, 1)$ ) is called quasi-m algebra (structure), if it satisfies the properties (qm-U), (U1). (qm1') Any commutative algebra (structure) $\mathcal{A}^{\prime}=$ $(A, \sigma)$ whose signature $\sigma$ contains $\odot, 1(\leq, \odot, 1$, respectively) is also called quasi-m algebra (structure), if it satisfies (qm-U) and (U1).

(qm1") Any commutative algebra (structure) $\mathcal{A}^{\prime \prime}=$ $(A, \tau)$ which is term equivalent to a quasi-m algebra (structure) $\mathcal{A}^{\prime}=(A, \sigma)$, is also called quasi-m algebra (structure).

A commutative quasi- $m$ unital magma is a commutative algebra $\mathcal{A}=(A, \odot, 1)$ verifying (qm-U) and (U1) [13]. Note that their class is the largest class of quasi-m algebras. Other examples of quasi-m algebras are the commutative quasi-m monoids, quasi-m groups [13], the quasi-MV algebras [15] (in fact quasi-m MV algebras, by [13]).

Remark 3.7 [13] (qm-U) is different of (U) if and only if $V_{U} \subset A$, and this is the basic, definable property of quasi-m algebras (quasi-m structures).

For every quasi-m algebra (structure) $\mathcal{A}$, the subset $V_{U}$ of $A$ will be called the regular-m set of $\mathcal{A}$ and will be denoted by $\operatorname{Rm}(A)$ :

$$
\operatorname{Rm}(A) \stackrel{\text { def. }}{=} V_{U} \subseteq A .
$$

The quasi-m algebra (structure) $\mathcal{A}$ is called qm-proper, if $R m(A) \neq A$ (i.e. (U) $\Longleftrightarrow(\mathrm{qm}-\mathrm{U})$ ); otherwise, $\mathcal{A}$ is a regular-m algebra (structure). The elements of $\operatorname{Rm}(A)$ are called the regular-m elements of $A$, the elements of $A \backslash R m(A)$ are called the quasi-m elements of $A$.

Theorem $3.8[13]$ Let $\mathcal{A}=(A, \odot, 1)$ be a qm-proper quasi-m algebra. Then, $\mathcal{R} m(\mathcal{A})=(\operatorname{Rm}(A), \odot, 1)$ is a regular-m algebra.

In a qm-proper quasi-m algebra, we shall call quasi$m$ property, a property that is dependent of (qm-U), and we shall call regular-m property, a property that is independent of $(\mathrm{qm}-\mathrm{U})$.

\subsubsection{Quasi-m Boolean algebras}

A quasi-m Boolean algebra is an algebra

$$
\left(A, \wedge, \vee,^{-}, 0,1\right)
$$

of type $(2,2,1,0,0)$ verifying: for all $x, y, z \in A$,

$\begin{array}{ll}\text { (UW1) } & 1 \wedge 1=1, \\ \text { (UV0) } & 0 \vee 0=0 ; \\ \text { (qm-Wid) } & x \wedge x=1 \wedge x, \\ \text { (qm-Vid) } & x \vee x=0 \vee x ; \\ \text { (mWcomm) } & x \wedge y=y \wedge x, \\ \text { (mVcomm) } & x \vee y=y \vee x ; \\ \text { (mWass) } & x \wedge(y \wedge z)=(x \wedge y) \wedge z, \\ \text { (mVass) } & x \vee(y \vee z)=(x \vee y) \vee z ; \\ \text { (qm-Abs1) } & x \vee(x \wedge y)=1 \wedge x, \\ \text { (qm-Abs2) } & x \wedge(x \vee y)=0 \vee x ;\end{array}$




$\begin{array}{ll}(\mathrm{IdU}) & 1 \wedge x=0 \vee x ; \\ (\mathrm{qm}-\mathrm{UW}) & 1 \wedge(x \wedge y)=x \wedge y, \\ (\mathrm{qm}-\mathrm{UV}) & 0 \vee(x \vee y)=x \vee y ; \\ (\mathrm{mDis} 1) & x \wedge(y \vee z)=(x \wedge y) \vee(x \wedge z), \\ (\mathrm{mDis} 2) & x \vee(y \wedge z)=(x \vee y) \wedge(x \vee z) ; \\ (\mathrm{mWx}) & x \wedge x^{-}=0, \\ (\mathrm{mVx}) & x \vee x^{-}=1 ; \\ (\mathrm{DN}) & \left(x^{-}\right)^{-}=x ; \\ (\mathrm{dfV}) & x \vee y=\left(x^{-} \wedge y^{-}\right)^{-} ; \\ (\mathrm{qm}-\overline{U W(1 \wedge x})) & 1 \wedge(1 \wedge x)^{-}=(1 \wedge x)^{-}, \\ (\mathrm{qm}-\overline{U V(0 \vee x})) & 0 \vee(0 \vee x)^{-}=(0 \vee x)^{-}\end{array}$

Note that a quasi-m Boolean algebra (that can be defined equivalently as an algebra $\left(A, \wedge,{ }^{-}, 1\right)$ of type $(2,1,0))$ is a quasi-m algebra, since (qm-UW), (UW1), and the dual (qm-UV), (UV1), hold. Note also that, if (U) (here (UW) and (UV)) holds, then any quasi-m Boolean algebra is a Boolean algebra.

Theorem 3.9 Let $\mathcal{A}=\left(A, \wedge, \vee,{ }^{-}, 0,1\right)$ be a qmproper quasi-m Boolean algebra. Then,

$$
\mathcal{R} m(\mathcal{A})=\left(\operatorname{Rm}(A), \wedge, \vee^{-}, 0,1\right)
$$

is a Boolean algebra.

Proposition 3.10 Let $\left(A, \wedge, \vee,{ }^{-}, 0,1\right)$ be a quasi-m Boolean algebra. We have:

(i) $\left(x \wedge y^{-}\right)^{-}=x^{-} \vee y$,

(ii) (De Morgan laws) $(x \wedge y)^{-}=x^{-} \vee y^{-}$and $(x \vee y)^{-}=x^{-} \wedge y^{-}$

(iii) $0^{-}=1$ and $1^{-}=0$,

(iv) $x \vee 1=1$ and $x \wedge 0=0$,

$(v)=(q m-N e g)(x \wedge 1)^{-}=x^{-} \wedge 1$ and $(x \vee 0)^{-}=x^{-} \vee 0$.

\subsection{Quasi-i-Boolean algebras vs. quasi-m Boolean algebras}

The quasi-i-Boolean algebras are categorically equivalent to the quasi-m Boolean algebras just as the quasiWajsberg algebras are categorically equivalent to the quasi-m MV algebras, namely we have:

Theorem 3.11 (See Theorem 2.3) bra

(1) Let $\mathcal{A}=\left(A, \rightarrow,{ }^{-}, 1\right)$ be a quasi-i-Boolean alge-

Define $\Phi(\mathcal{A}) \stackrel{\text { def. }}{=}\left(A, \wedge, \vee,{ }^{-}, 0,1\right)$ as follows: for every $x, y \in A, \quad x \wedge y \stackrel{\text { def. }}{=}\left(x \rightarrow y^{-}\right)^{-}$, $x \vee y \stackrel{\text { def. }}{=}\left(x^{-} \wedge y^{-}\right)^{-}=x^{-} \rightarrow y, \quad 0 \stackrel{\text { def. }}{=} 1^{-}$.

Then, $\Phi(\mathcal{A})$ is a quasi-m Boolean algebra.

(1') Conversely, let $\mathcal{A}=(A, \wedge, \vee,-, 0,1)$ be a quasi-m Boolean algebra.

Define $\Psi(\mathcal{A}) \stackrel{\text { def. }}{=}(A, \rightarrow,-, 1)$ as follows: for every $x, y \in A, \quad x \rightarrow y \stackrel{\text { def. }}{=}\left(x \wedge y^{-}\right)^{-} \stackrel{(i)}{=} x^{-} \vee y$.

Then, $\Psi(\mathcal{A})$ is a quasi-i-Boolean algebra.

(2) The mappings $\Phi$ and $\Psi$ are mutually inverse.
Proof. (1): First note that we have: (a) $1 \wedge x=1 \rightarrow x$ and (b) $0 \vee x=1 \rightarrow x$. Indeed, $1 \wedge x=\left(1 \rightarrow x^{-}\right)^{-} \stackrel{(\beta)}{=}\left((1 \rightarrow x)^{-}\right)^{-} \stackrel{(D N)}{=}$ $1 \rightarrow x$, thus (a) holds.

$0 \vee x=0^{-} \rightarrow x \stackrel{(N e g 0-1)}{=} 1 \rightarrow x$, thus (b) holds too.

$(\mathrm{UW} 1): 1 \wedge 1=\left(1 \rightarrow 1^{-}\right)^{-} \stackrel{(N e g 1-0)}{=}$

$(1 \rightarrow 0)^{-} \stackrel{(q-N e g)}{=} 0 \rightarrow 0 \stackrel{(R e)}{=} 1$ and

$(\mathrm{UV0}): 0 \vee 0=0^{-} \rightarrow 0 \stackrel{(N e g 0-1)}{=} 1 \rightarrow 0 \stackrel{(q-N e g)}{=}$ $(1 \rightarrow 1)^{-\stackrel{(R e)}{=}} 1^{-\stackrel{(N e g 1-0)}{=} 0 .}$

(qm-Wid): $x \wedge x=\left(x \rightarrow x^{-}\right)^{-} \stackrel{(q-I 2)}{=}$ $\left(x \rightarrow\left(1 \rightarrow x^{-}\right)\right)^{-} \stackrel{(\beta)}{=}\left[x \rightarrow(1 \rightarrow x)^{-}\right]^{-} \stackrel{(q-N e g 6)}{=}$ $\left((1 \rightarrow x)^{-}\right)^{-} \stackrel{(D N)}{=} 1 \rightarrow x \stackrel{(a)}{=} 1 \wedge x$ and

(qm-Vid): $x \vee x=\left(x^{-} \wedge x^{-}\right)^{-} \stackrel{(q m-W i d)}{=}$ $\left(1 \wedge x^{-}\right)^{-} \stackrel{(N e g 0-1)}{=}\left(0^{-} \wedge x^{-}\right)^{-}=0 \vee x$.

$(\mathrm{mWcomm}): x \wedge y=\left(x \rightarrow y^{-}\right)^{-} \stackrel{(N e g 3)}{=}$ $\left(y \rightarrow x^{-}\right)^{-}=y \wedge x$ and

$(\mathrm{mVcomm}): x \vee y=\left(x^{-} \wedge y^{-}\right)^{-}=\left(y^{-} \wedge x^{-}\right)^{-}=$ $y \vee x$.

(mWass): $x \wedge(y \wedge z)=x \wedge\left(y \rightarrow z^{-}\right)^{-}=$ $\left[x \rightarrow\left(\left(y \rightarrow z^{-}\right)^{-}\right)^{-}\right]^{-} \stackrel{(D N)}{=}\left[x \rightarrow\left(y \rightarrow z^{-}\right)\right]^{-}$and

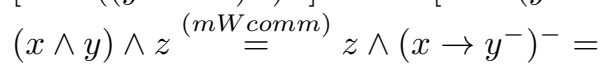
$\left[z \rightarrow\left(\left(x \rightarrow y^{-}\right)^{-}\right)^{-}\right]^{-} \stackrel{(D N)}{=}\left[z \rightarrow\left(x \rightarrow y^{-}\right)\right]^{-} \stackrel{(E x)}{=}$ $\left[x \rightarrow\left(z \rightarrow y^{-}\right)\right]^{-} \stackrel{\left(N_{e g} 3\right)}{=}\left[x \rightarrow\left(y \rightarrow z^{-}\right)\right]^{-}$, hence $x \wedge(y \wedge z)=(x \wedge y) \wedge z$.

(mVass): $x \vee(y \vee z)=x \vee\left(y^{-} \wedge z^{-}\right)^{-}=$ $\left[x^{-} \wedge\left(\left(y^{-} \wedge z^{-}\right)^{-}\right)^{-}\right]^{-} \stackrel{(D N)}{=}\left[x^{-} \wedge\left(y^{-} \wedge z^{-}\right)\right]^{-} \stackrel{(m \text { Wass })}{=}$ $\left[\left(x^{-} \wedge y^{-}\right) \wedge z^{-}\right]^{-} \stackrel{(D N)}{=}\left[\left(\left(x^{-} \wedge y^{-}\right)^{-}\right)^{-} \wedge z^{-}\right]^{-}=$ $\left[(x \vee y)^{-} \wedge z^{-}\right]^{-}=(x \vee y) \vee z$.

$(\mathrm{qm}-\mathrm{Abs} 1): x \vee(x \wedge y) \stackrel{(m V \operatorname{comm})}{=}(x \wedge y) \vee x=$ $(x \wedge y)^{-} \rightarrow x=\left(\left(x \rightarrow y^{-}\right)^{-}\right)^{-} \rightarrow x \stackrel{(D N)}{=}$ $\left(x \rightarrow y^{-}\right) \rightarrow x \stackrel{(q-i m p l)}{=} 1 \rightarrow x \stackrel{(a)}{=} 1 \wedge x$ and $(\mathrm{qm}-\mathrm{Abs} 2): x \wedge(x \vee y) \stackrel{(m W \text { comm })}{=}(x \vee y) \wedge x=$ $\left[\left(x^{-} \rightarrow y\right) \rightarrow x^{-}\right]^{-} \stackrel{(q-i m p l)}{=}\left(1 \rightarrow x^{-}\right)^{-}=1 \wedge x \stackrel{(a)}{=}$ $1 \rightarrow x \stackrel{(b)}{=} 0 \vee x$.

$(\mathrm{IdU}): 1 \wedge x \stackrel{(a)}{=} 1 \rightarrow x \stackrel{(b)}{=} 0 \vee x$.

$(\mathrm{qm}-\mathrm{UW}): 1 \wedge(x \wedge y)=1 \wedge\left(x \rightarrow y^{-}\right)^{-}=$ $\left[1 \rightarrow\left(\left(x \rightarrow y^{-}\right)^{-}\right)^{-}\right]^{-} \stackrel{(D N)}{=}\left[1 \rightarrow\left(x \rightarrow y^{-}\right)\right]^{-} \stackrel{(q-M)}{=}$ $\left(x \rightarrow y^{-}\right)^{-}=x \wedge y$ and

$(\mathrm{qm}-\mathrm{UV}): 0 \vee(x \vee y)=0 \vee\left(x^{-} \rightarrow y\right)=$ $0^{-} \rightarrow\left(x^{-} \rightarrow y\right) \stackrel{(N e g 0-1)}{=} 1 \rightarrow\left(x^{-} \rightarrow y\right) \stackrel{(q-M)}{=}$ $x^{-} \rightarrow y=x \vee y$.

(mDis1): If $x \leq y \stackrel{\text { def. }}{\Longleftrightarrow} x \rightarrow y=1$, then we shall prove:

(c) $(x \wedge z) \vee(y \wedge z) \leq(x \vee y) \wedge z$ and

(d) $(x \vee y) \wedge z \leq(x \wedge z) \vee(y \wedge z)$.

To prove (c): since $x \wedge z \leq z$ and $y \wedge z \leq z$, then 
$(x \wedge z) \vee(y \wedge z) \leq z \vee z \stackrel{(q m-V i d)}{=} 0 \vee z$ and since $x \wedge z \leq x$ and $y \wedge z \leq y$, then $(x \wedge z) \vee(y \wedge z) \leq x \vee y$, by $(\mathrm{j})$ and (jj); then,

$(x \wedge z) \vee(y \wedge z) \stackrel{(q m-U V)}{=} 0 \vee[(x \wedge z) \vee(y \wedge z)] \stackrel{(I d U)}{=}$ $1 \wedge[(x \wedge z) \vee(y \wedge z)] \stackrel{(q m-W i d)}{=}$

$[(x \wedge z) \vee(y \wedge z)] \wedge[(x \wedge z) \vee(y \wedge z)] \stackrel{(j j)}{\leq}(0 \vee z) \wedge(x \vee y) \stackrel{(I d U)}{=}$ $(1 \wedge z) \wedge(x \vee y) \stackrel{(m W a s s)}{=} 1 \wedge[z \wedge(x \vee y)] \stackrel{(q m-U W)}{=}$

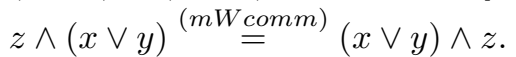

To prove $(d)$, first we prove

$$
x \vee y \leq z \rightarrow[(x \wedge z) \vee(y \wedge z)] .
$$

Indeed, since $z \rightarrow[(x \wedge z) \vee(y \wedge z)] \stackrel{(D N),(m W \text { comm })}{=}$ $z \rightarrow\left[\left(z \rightarrow x^{-}\right) \rightarrow\left(y \rightarrow z^{-}\right)^{-}\right] \stackrel{(E x)}{=}$

$\left(z \rightarrow x^{-}\right) \rightarrow\left[z \rightarrow\left(y \rightarrow z^{-}\right)^{-}\right]$

then (1) is equivalent to

$$
x \vee y \leq\left(z \rightarrow x^{-}\right) \rightarrow\left[z \rightarrow\left(y \rightarrow z^{-}\right)^{-}\right] .
$$

Now we prove

$$
y \leq z \rightarrow\left(y \rightarrow z^{-}\right)^{-} .
$$

Indeed, $y \stackrel{\left(D^{\prime}\right)}{\leq}\left(y \rightarrow z^{-}\right) \rightarrow z^{-\stackrel{(D N 2),(D N)}{=}}$

$z \rightarrow\left(y \rightarrow z^{-}\right)^{-}$, hence $(3)$ holds.

From (3), by $\left(^{*},\right)$, we obtain:

$\left(z \rightarrow x^{-}\right) \rightarrow y \leq\left(z \rightarrow x^{-}\right) \rightarrow\left[z \rightarrow\left(y \rightarrow z^{-}\right)^{-}\right]$

and, by $\left(\mathrm{K}^{\prime}\right)$, we obtain: $y \leq\left(z \rightarrow x^{-}\right) \rightarrow y$; it follows by $\left(\operatorname{Tr}^{\prime}\right)$ that

$$
y \leq\left(z \rightarrow x^{-}\right) \rightarrow\left[z \rightarrow\left(y \rightarrow z^{-}\right)^{-}\right] .
$$

By (B'), we obtain:

$x^{-} \rightarrow\left(y \rightarrow z^{-}\right)^{-} \leq\left(z \rightarrow x^{-}\right) \rightarrow\left[z \rightarrow\left(y \rightarrow z^{-}\right)^{-}\right]$ and by (K'), (DN2), we obtain:

$x \leq\left(y \rightarrow z^{-}\right) \rightarrow x=x^{-} \rightarrow\left(y \rightarrow z^{-}\right)^{-}$.

It follows, by ( $\left.\mathrm{Tr}^{\prime}\right)$, that

$$
x \leq\left(z \rightarrow x^{-}\right) \rightarrow\left[z \rightarrow\left(y \rightarrow z^{-}\right)^{-}\right] .
$$

From (4) and (5), we obtain, by (jj):

$$
x \vee y \leq
$$$$
\left(\left(z \rightarrow x^{-}\right) \rightarrow\left[z \rightarrow\left(y \rightarrow z^{-}\right)^{-}\right]\right) \vee
$$$$
\left(\left(z \rightarrow x^{-}\right) \rightarrow\left[z \rightarrow\left(y \rightarrow z^{-}\right)^{-}\right]\right) \stackrel{(q m-V i d)}{=}
$$$$
0 \vee\left(\left(z \rightarrow x^{-}\right) \rightarrow\left[z \rightarrow\left(y \rightarrow z^{-}\right)^{-}\right]\right) \stackrel{(b)}{=}
$$$$
1 \rightarrow\left(\left(z \rightarrow x^{-}\right) \rightarrow\left[z \rightarrow\left(y \rightarrow z^{-}\right)^{-}\right]\right) \stackrel{(q-M)}{=}
$$$$
\left(z \rightarrow x^{-}\right) \rightarrow\left[z \rightarrow\left(y \rightarrow z^{-}\right)^{-}\right] \text {, }
$$

hence (2) holds, hence (1) holds.

Now, since (1) means

$(x \vee y) \rightarrow(z \rightarrow[(x \wedge z) \vee(y \wedge z)])=1$,

it follows, by (WEx), that

$[(x \vee y) \wedge z] \rightarrow[(x \wedge z) \vee(y \wedge z)]=1$, i.e. (d) holds.

By (c), (d) and (q-An), ( $\beta),(\mathrm{q}-\mathrm{M})$, we obtain:

$(x \vee y) \wedge z=\left((x \vee y) \rightarrow z^{-}\right)^{-} \stackrel{(q-M)}{=}$ $\left[1 \rightarrow\left((x \vee y) \rightarrow z^{-}\right)\right]^{-} \stackrel{(\beta)}{=} 1 \rightarrow\left[(x \vee y) \rightarrow z^{-}\right]^{-}=$

$1 \rightarrow[(x \vee y) \wedge z] \stackrel{(q-A n)}{=} 1 \rightarrow[(x \wedge z) \vee(y \wedge z)]=$ $1 \rightarrow\left[(x \wedge z)^{-} \rightarrow(y \wedge z)\right] \stackrel{(q-M)}{=}(x \wedge z)^{-} \rightarrow(y \wedge z)=$ $(x \wedge z) \vee(y \wedge z)$, i.e. (mDis1) holds. The second part, (mDis2), has a similar proof.

$(\mathrm{mWx}): x \wedge x^{-}=\left(x \rightarrow\left(x^{-}\right)^{-}\right)^{-} \stackrel{(D N)}{=}$ $(x \rightarrow x)^{-} \stackrel{(R e)}{=} 1^{-} \stackrel{(N e g 1-0)}{=} 0$ and

$(\mathrm{mVx}): x \vee x^{-}=x^{-} \rightarrow x^{-\stackrel{(R e)}{=}} 1$.

$(\mathrm{DN})$ : By hypothesis. (dfV): By definition.

$(\mathrm{qm}-\overline{U W(1 \wedge x})): 1 \wedge(1 \wedge x)^{-} \stackrel{(a)}{=}$

$1 \rightarrow(1 \rightarrow x)^{-} \stackrel{(q-\overline{M(1 \rightarrow x)})}{=}(1 \rightarrow x)^{-} \stackrel{(a)}{=}(1 \wedge x)^{-}$and

$(\mathrm{qm}-\overline{U V(0 \vee x})): 0 \vee(0 \vee x)^{-} \stackrel{(b)}{=}$ $1 \rightarrow(1 \rightarrow x)^{-} \stackrel{(q-\overline{M(1 \rightarrow x)})}{=}(1 \rightarrow x)^{-} \stackrel{(b)}{=}(0 \vee x)^{-}$.

Thus, $\Phi(\mathcal{A})$ is a quasi-m Boolean algebra.

$\left(1^{\prime}\right)$ : First note that we have:

(e) $1 \rightarrow x=0 \vee x \stackrel{(I d U)}{=} 1 \wedge x$.

Indeed, $1 \rightarrow x=\left(1 \wedge x^{-}\right)^{-} \stackrel{(i i i)}{=}\left(0^{-} \wedge x^{-}\right)^{-} \stackrel{(d f V)}{=} 0 \vee x$.

$(\mathrm{K}): x \rightarrow(y \rightarrow x)=x^{-} \vee(y \rightarrow x)=$

$x^{-} \vee\left(y^{-} \vee x\right)^{(m V \text { comm }),(m V a s s)}\left(x \vee x^{-}\right) \vee y^{-} \stackrel{(m V x)}{=}$

$1 \vee y^{-\stackrel{(i v)}{=}} 1$

(pimpl-1): $[x \rightarrow(y \rightarrow z)] \rightarrow[(x \rightarrow y) \rightarrow(x \rightarrow z)]$ $=\left[x^{-} \vee\left(y^{-} \vee z\right)\right]^{-} \vee\left[\left(x^{-} \vee y\right)^{-} \vee\left(x^{-} \vee z\right)\right] \stackrel{(D N),(i i)}{=}$ $\left[x \wedge\left(y \wedge z^{-}\right)\right] \vee\left[\left(x \wedge y^{-}\right) \vee x^{-} \vee z\right] \stackrel{(m D i s 2)}{=}$

$\left[x \wedge y \wedge z^{-}\right] \vee\left[\left(x \vee x^{-} \vee z\right) \wedge\left(y^{-} \vee x^{-} \vee z\right)\right] \stackrel{(m V x),(i v)}{=}$ $\left[x \wedge y \wedge z^{-}\right] \vee\left[1 \wedge\left(y^{-} \vee x^{-} \vee z\right)\right] \stackrel{(I d U)}{=}$

$\left[x \wedge y \wedge z^{-}\right] \vee\left[0 \vee\left(y^{-} \vee x^{-} \vee z\right)\right] \stackrel{(q m-U V)}{=}$

$\left[x \wedge y \wedge z^{-}\right] \vee\left[y^{-} \vee x^{-} \vee z\right] \stackrel{(i i)}{=}$

$\left[x \wedge y \wedge z^{-}\right] \vee\left[y \wedge x \wedge z^{-}\right]^{-} \stackrel{(m V x)}{=} 1$.

$(\mathrm{DN} 1):\left(y^{-} \rightarrow x^{-}\right) \rightarrow(x \rightarrow y)=$

$\left(\left(y^{-}\right)^{-} \vee x^{-}\right)^{-} \vee\left(x^{-} \vee y\right) \stackrel{(D N)}{=}$

$\left(y \vee x^{-}\right)^{-} \vee\left(x^{-} \vee y\right) \stackrel{(m V x)}{=} 1$.

(q-An): $x \rightarrow y=1=y \rightarrow x$ means $x^{-} \vee y=1=$ $y^{-} \vee x$, hence $0=y \wedge x^{-}$, by (DN), (ii), (iii). Then,

$1 \rightarrow x=1^{-} \vee x \stackrel{(i i i)}{=} 0 \vee x=$

$\left(y \wedge x^{-}\right) \vee x \stackrel{(m V c o m m),(m D i s 2)}{=}(y \vee x) \wedge\left(x^{-} \vee x\right) \stackrel{(m V x)}{=}$ $(y \vee x) \wedge 1=(y \vee x) \wedge\left(x^{-} \vee y\right)=(y \vee x) \wedge\left(y \vee x^{-}\right) \stackrel{(m D i s 2)}{=}$ $y \vee\left(x \wedge x^{-}\right) \stackrel{(m W x)}{=} y \vee 0 \stackrel{(e)}{=} 1 \rightarrow y$.

$(\mathrm{q}-\mathrm{M}): 1 \rightarrow(x \rightarrow y)=1^{-} \vee\left(x^{-} \vee y\right) \stackrel{(i i i)}{=}$ $0 \vee\left(x^{-} \vee y\right) \stackrel{q m-U V)}{=} x^{-} \vee y=x \rightarrow y$.

(DN): By hypothesis.

$(\mathrm{q}-\overline{M(1 \rightarrow x)}): 1 \rightarrow(1 \rightarrow x)^{-}=1^{-} \vee\left(1^{-} \vee x\right)^{-} \stackrel{(i i i)}{=}$ $0 \vee(0 \vee x)^{-(q m-\overline{U V(0 \vee x)})}(0 \vee x)^{-}=\left(1^{-} \vee x\right)^{-}=$ $(1 \rightarrow x)^{-}$.

Thus, $\Psi(\mathcal{A})$ is a quasi-i-Boolean algebra. 
(2): Let

$\left(A, \rightarrow^{-}, 1\right) \stackrel{\Phi}{\longrightarrow}\left(A, \wedge, \vee,^{-}, 0,1\right) \stackrel{\Psi}{\longrightarrow}\left(A, \Rightarrow,,^{-}, 1\right)$.

Then, for all $x, y \in A$, we have:

$x \Rightarrow y=x^{-} \vee y=\left(x^{-}\right)^{-} \rightarrow y \stackrel{(D N)}{=} x \rightarrow y$,

hence $\Psi \circ \Phi=1_{(A, \rightarrow,-, 1)}$.

Conversely, let

$\left(A, \wedge, \vee,^{-}, 0,1\right) \stackrel{\Psi}{\rightarrow}(A, \rightarrow,-, 1) \stackrel{\Phi}{\rightarrow}\left(A, \wedge, \bigvee,^{-}, \mathbf{0}, 1\right)$

Then, for all $x, y \in A$, we have:

$x \wedge y=\left(x \rightarrow y^{-}\right)^{-}=\left(x^{-} \vee y^{-}\right)^{-} \stackrel{(i i),(D N)}{=} x \wedge y$,

$x \bigvee y=x^{-} \rightarrow y=\left(x^{-}\right)^{-} \vee y=x \vee y, \mathbf{0}=1^{-} \stackrel{(\text { iii })}{=} 0$, hence $\Phi \circ \Psi=1_{(A, \wedge, \vee,-, 0,1)}$.

\subsection{Examples}

Consider the standard i-Boolean algebra

$$
\mathcal{L}_{2}=\left(L_{2}=\{0,1\}, \rightarrow,-, 1\right)
$$

represented by the Hasse diagram given in Figure 1 and with the tables of $\rightarrow$ and ${ }^{-}$(involutive negation) recalled below:

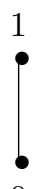

0

$$
\mathcal{L}_{2}
$$

Boolean algebra

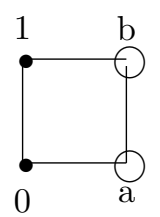

$\mathcal{L}_{2}^{1}$

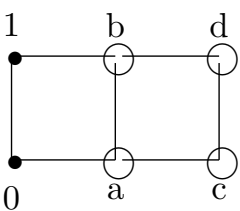

$\mathcal{L}_{2}^{2^{1,2}}$
quasi-i-Boolean algebras

Figure 1: The Boolean algebra $\mathcal{L}_{2}$ and the quasi-iBoolean algebras $\mathcal{L}_{2}^{1}, \mathcal{L}_{2}^{2^{1,2}}$

\begin{tabular}{cc|cc} 
& $\rightarrow$ & $\mathbf{0}$ & $\mathbf{1}$ \\
\cline { 2 - 4 } $\mathcal{L}_{2}$ & $\mathbf{0}$ & $\mathbf{1}$ & $\mathbf{1}$
\end{tabular},

\begin{tabular}{c|c}
$x$ & $x^{-}(=x \rightarrow 0)$ \\
\hline $\mathbf{0}$ & $\mathbf{1}$ \\
$\mathbf{1}$ & $\mathbf{0}$
\end{tabular}.

By Theorem 2.3, the standard i-Boolean algebra $\mathcal{L}_{2}$ is term-equivalent to the standard Boolean algebra

$$
\mathcal{L}_{2}^{m}=\left(L_{2}=\{0,1\}, \wedge, \vee,^{-}, 0,1\right)
$$

represented by the same Hasse diagram given in Figure 1 and with the tables of $\wedge, \vee$ given below and the same table of ${ }^{-}$(involutive negation):

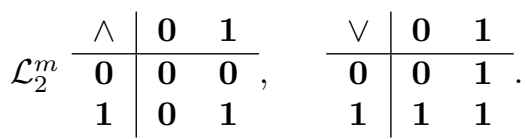

We can build an infinity of quasi-i-Boolean algebras whose regular algebra be the standard i-Boolean algebra $\mathcal{L}_{2}$, by [10]. We present two examples.

\subsubsection{Example 1}

By adding two quasi-elements, $a, b$, to the above regular elements 0,1 , such that $a \| 0$ and $b \| 1$ (see [10]), we obtain the quasi-i-Boolean algebra

$$
\mathcal{L}_{2}^{1}=\left(L_{2}^{1}=\{0, a, b, 1\}, \rightarrow^{1},{ }^{-}, 1\right),
$$

represented by the quasi-Hasse diagram (see [10]) given also in Figure 1 and with the following tables of $\rightarrow^{1}$ and ${ }^{-}$(involutive quasi-negation):

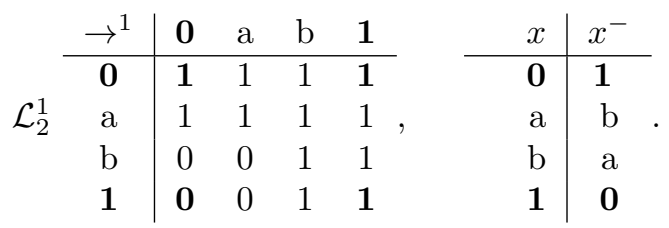

Note that the line/column of $a(b)$ coincides with the line/column of 0 ( 1 , respectively).

By Theorem 3.11, the quasi-i-Boolean algebra $\mathcal{L}_{2}^{1}$

\begin{tabular}{|c|c|c|c|c|c|c|c|c|c|}
\hline \multirow{5}{*}{$\mathcal{L}_{2}^{1 m}$} & 0 & $\mathrm{a}$ & $\mathrm{b}$ & 1 & $\vee^{1}$ & 0 & $\mathrm{a}$ & $\mathrm{b}$ & 1 \\
\hline & 0 & 0 & 0 & 0 & 0 & 0 & 0 & 1 & 1 \\
\hline & 0 & 0 & 0 & 0 & $\mathrm{a}$ & 0 & 0 & 1 & 1 \\
\hline & 0 & 0 & 1 & 1 & $\mathrm{~b}$ & 1 & 1 & 1 & 1 \\
\hline & 0 & 0 & 1 & 1 & 1 & 1 & 1 & 1 & 1 \\
\hline
\end{tabular}
is term-equivalent to the quasi-m Boolean algebra

$$
\mathcal{L}_{2}^{1 m}=\left(L_{2}^{1}=\{0, a, b, 1\}, \wedge^{1}, \vee^{1},-, 0,1\right),
$$

with the tables of $\wedge^{1}, \vee^{1}$ given below and the same table of ${ }^{-}$(involutive quasi-m negation, this time):

Note that the line/column of $a(b)$ coincides with the line/column of 0 ( 1 , respectively).

Note that $\mathcal{R}\left(\mathcal{L}_{2}^{1}\right)=\mathcal{L}_{2}$ and $\mathcal{R} m\left(\mathcal{L}_{2}^{1 m}\right)=\mathcal{L}_{2}^{m}$.

\subsubsection{Example 2}

By adding four quasi-elements, $a, b, c, d$, to the above regular elements 0,1 , such that $c\|a\| 0$ and $d\|b\| 1$ (see [10]), we obtain two quasi-i-Boolean algebras:

$\mathcal{L}_{2}^{2^{1}}=\left(L_{2}^{2}=\{0, a, b, c, d, 1\}, \rightarrow^{2},{ }^{-1}, 1\right)$ and $\mathcal{L}_{2}^{2}=\left(L_{2}^{2}=\{0, a, b, c, d, 1\}, \rightarrow^{2},{ }^{2}{ }^{2}, 1\right)$, respectively,

\begin{tabular}{|c|c|c|c|c|c|c|c|c|c|}
\hline$\rightarrow^{2}$ & 0 & $\mathrm{a}$ & $\mathrm{b}$ & $\mathrm{c}$ & $\mathrm{d}$ & 1 & $x$ & $x^{-^{1}}$ & $x^{-^{2}}$ \\
\hline 0 & 1 & 1 & 1 & 1 & 1 & 1 & 0 & 1 & 1 \\
\hline $\mathrm{a}$ & 1 & 1 & 1 & 1 & 1 & 1 & $\mathrm{a}$ & b & d \\
\hline $\mathrm{b}$ & 0 & 0 & 1 & 0 & 1 & 1 & $\mathrm{~b}$ & $\mathrm{a}$ & $\mathrm{c}$ \\
\hline $\mathrm{c}$ & 1 & 1 & 1 & 1 & 1 & 1 & $\mathrm{c}$ & d & b \\
\hline $\mathrm{d}$ & 0 & 0 & 1 & 0 & 1 & 1 & $\mathrm{~d}$ & $\mathrm{c}$ & $\mathrm{a}$ \\
\hline 1 & 0 & 0 & 1 & 0 & 1 & 1 & 1 & 0 & 0 \\
\hline
\end{tabular}
(with the same table of $\rightarrow^{2}$, but with different involutive quasi-negations),

represented by the quasi-Hasse diagram (see [10]) given also in Figure 1 and with the following tables of $\rightarrow^{2}$ and ${ }^{-1},{ }^{-2}$ : 
Note that the lines/columns of $a, c(b, d)$ coincide with the line/column of 0 (1, respectively).

By Theorem 3.11, the quasi-i-Boolean algebras $\mathcal{L}_{2}^{2^{1,2}}$ are term-equivalent to the quasi-m Boolean algebras:

$\mathcal{L}_{2}^{2^{1 m}}=\left(L_{2}^{2}=\{0, a, b, c, d, 1\}, \wedge^{2}, \vee^{2},{ }^{-1}, 0,1\right)$ and $\mathcal{L}_{2}^{2^{2 m}}=\left(L_{2}^{2}=\{0, a, b, c, d, 1\}, \wedge^{2}, \vee^{2},{ }^{2}, 0,1\right)$, respectively, with the tables of $\wedge^{2}, \vee^{2}$ given below and the same tables of ${ }^{-1}$ and ${ }^{-2}$ (involutive quasi-m negations, this time):

\begin{tabular}{cc|cccccc} 
& $\wedge^{2}$ & $\mathbf{0}$ & $\mathrm{a}$ & $\mathrm{b}$ & $\mathrm{c}$ & $\mathrm{d}$ & $\mathbf{1}$ \\
\hline \multirow{4}{*}{$\mathcal{L}_{2}^{2^{1,2 m}}$} & $\mathbf{0}$ & $\mathbf{0}$ & 0 & 0 & 0 & 0 & $\mathbf{0}$ \\
$\mathrm{a}$ & 0 & 0 & 0 & 0 & 0 & 0 \\
$\mathrm{~b}$ & 0 & 0 & 1 & 0 & 1 & 1 \\
& $\mathrm{c}$ & 0 & 0 & 0 & 0 & 0 & 0 \\
$\mathrm{~d}$ & 0 & 0 & 1 & 0 & 1 & 1 \\
$\mathbf{1}$ & $\mathbf{0}$ & 0 & 1 & 0 & 1 & $\mathbf{1}$ \\
& \\
$\vee^{2}$ & $\mathbf{0}$ & $\mathrm{a}$ & $\mathrm{b}$ & $\mathrm{c}$ & $\mathrm{d}$ & $\mathbf{1}$ \\
\hline $\mathbf{0}$ & $\mathbf{0}$ & 0 & 1 & 0 & 1 & $\mathbf{1}$ \\
$\mathrm{a}$ & 0 & 0 & 1 & 0 & 1 & 1 \\
$\mathrm{~b}$ & 1 & 1 & 1 & 1 & 1 & 1 \\
$\mathrm{c}$ & 0 & 0 & 1 & 0 & 1 & 1 \\
$\mathrm{~d}$ & 1 & 1 & 1 & 1 & 1 & 1 \\
$\mathbf{1}$ & $\mathbf{1}$ & 1 & 1 & 1 & 1 & $\mathbf{1}$
\end{tabular}.

Note that the lines/columns of $a, c(b, d)$ coincide with the line/column of 0 (1, respectively).

Note that $\mathcal{R}\left(\mathcal{L}_{2}^{2^{1,2}}\right)=\mathcal{L}_{2}$ and $\mathcal{R} m\left(\mathcal{L}_{2}^{2^{1,2 m}}\right)=\mathcal{L}_{2}^{m}$.

Other examples will be presented in the book in preparation, on quasi-algebras vs. quasi-m algebras.

\section{References}

[1] F. Bou, F. Paoli, A. Ledda, H. Freytes, On some properties of quasi-MV algebras and $\sqrt{{ }^{\prime}}$ quasi-MV algebras. Part II, Soft Comput., 2008, 12 (4), pp. 341-352.

[2] F. Bou, F. Paoli, A. Ledda, M. Spinks, R. Giuntini, The Logic of Quasi-MV algebras, Journal of Logic and Computation, 2010, 20, 2, pp. 619-643.

[3] G. Georgescu, A. Iorgulescu, Logică matematică (Romanian), Editura ASE (Academy of Economic Studies Publishing House), Bucharest, Romania, 2010.

[4] R. Giuntini, Weakly linear quantum MV algebras, Algebra Universalis, 2005, 53, 1, pp. 45-72.

[5] T. Kowalski, F. Paoli, M. Spinks, Quasisubtractive varieties, J. Symb. Log., 2011, 76 (4), pp. $1261-1286$
[6] T. Kowalski, F. Paoli, On some properties of quasi-MV algebras and $\sqrt{{ }^{\prime}}$ quasi-MV algebras. Part III, Rep. Math. Logic, 2010, 45, pp. 1611999.

[7] A. Iorgulescu, Algebras of logic as BCK algebras, Academy of Economic Studies Press, Bucharest, Romania, 2008.

[8] A. Iorgulescu, Asupra algebrelor Booleene (Romanian), Revista de logică http://egovbus.net/rdl, 25.01.2009, 1-25.

[9] A. Iorgulescu, New generalizations of BCI, BCK and Hilbert algebras - Parts I, II, (Dedicated to Dragoş Vaida) J. of Mult.-Valued Logic and Soft Computing, 2016, Vol. 27, No. 4, pp. 353-406, 407-456 (a previous version available from December 6, 2013, at http://arxiv.org/abs/1312.2494).

[10] A. Iorgulescu, Quasi-algebras versus regular algebras - Part I, Scientific Annals of Computer Science, 2015, vol. 25 (1), pp. 1-43.

[11] A. Iorgulescu, Quasi-algebras vs. regular algebras - Part II, 2015, Preprint nr. 3/2017 (http : //imar.ro/ increst/2017/3_2017.pdf).

[12] A. Iorgulescu, Quasi-algebras vs. regular algebras - Part III, 2015, Preprint nr. 4/2017 (http : //imar.ro/ increst/2017/4_2017.pdf).

[13] A. Iorgulescu, Implicative-groups vs. groups and generalizations, Matrix Rom, Bucharest, Romania, 2018.

[14] P. Jipsen, A. Ledda, F. PAoli, On some properties of quasi-MV algebras and $\sqrt{ }{ }^{\prime}$ quasi-MV algebras. Part IV, Rep. Math. Logic, 2013, 48, pp. $3-36$.

[15] A. Ledda, M. Konig, F. Paoli, R. GiunTINI, MV Algebras and Quantum Computation, Studia Logica, 2006, 82, 2, pp. 245-270.

[16] R. Padmanabhan, S. Rudeanu, Axioms for Lattices and Boolean Algebras, World Scientific, 2008 .

[17] F. Paoli, A. Ledda, R. Giuntini, H. Freytes, On some properties of quasi-MV algebras and $\sqrt{{ }^{\prime}}$ quasi-MV algebras. Part I, Rep. Math. Logic, 2009, 44, pp. 31-63. 